\section{Avaliação da onda V da audiometria de tronco cerebral de crianças reprovadas na triagem auditiva neonatal}

\author{
Viviane Camargo Marques ${ }^{1}$, Luis Miguel \\ Chiriboga Arteta', Elaine Soares ${ }^{2}$
}

Evaluation of the five wave from auditory brain response of children who not passed in neonatal screening

Resumo / Summary

á está bem estabelecido a importância do diagnóstico precoce das disacusias em neonatos. Muito se tem falado da triagem auditiva neonatal (TAN), mas pouco se comenta do encaminhamento e seguimento das crianças com emissão otoacústica (EOA) alterada. A audiometria de tronco cerebral (ABR) é a próxima etapa para o diagnóstico de disacusia nesta faixa etária. Objetivos: Os objetivos deste trabalho são avaliar a adesão ao programa de TAN e avaliar as ABRs das crianças reprovadas na TAN, concentrando a análise nos limiares obtidos e na latência da onda V. Tipo de Estudo: Clínico prospectivo. Material e Método: Análise de 75 ABRs de crianças atendidas no serviço de EOA da Maternidade de Campinas. Resultados: A maioria das crianças apresentou ABR com limiares e latências dentro dos valores normais. A alteração mais encontrada foi o aumento da latência da onda $V$, que pode ser decorrente da incompleta mielinização do sistema nervoso central, da presença de secreção em ouvido médio e de alterações retrococleares. Conclusão: Concluímos que o aumento da latência da onda $V$ é a alteração mais freqüente nas $A B R s ;$ que muitos pacientes se perdem durante a execução do protocolo de triagem auditiva, retardando ou impossibilitando o diagnóstico correto e precoce e que a maior parte das crianças reprovadas nas otoemissões acústicas têm limiar eletrofisiológico auditivo normal. $t$ is very well established the importance of early diagnostic of hearing loss. Everybody speaks about neonatal screening, but just a few people comment about the children who not passed on screening. The auditory brainstem response (ABR) is the next step of the diagnostic. The aim of this paper is evaluate the ABRs from children who not passed on screening, looking for the threshold and the latency of the five wave. Study Design: Clinical prospective. Material and Method: 75 ABR were analyzed. Results: A great number of exams was normal. The most frequently alteration was the increase of the latency of the wave $V$ that can be explained for the incomplete mielinization from the auditory pathway, otitis media or retrococlear alterations. Conclusions: We concluded that the increase of the latency of the $V$ wave is the mainly alteration on ABRs.

\footnotetext{
1 Médicos do serviço de otorrinolaringologia da Maternidade de Campinas.

${ }^{2}$ Fonoaudióloga do serviço de triagem auditiva neonatal da Maternidade de Campinas. Trabalho desenvolvido na Maternidade de Campinas.

Endereço para Correspondência: Dra. Viviane Camargo Marques - Rua Cristovão Colombo, 185/ 121 Campinas SP $13023-230$. Tel (0xx19) 3234-1017

Trabalho apresentado como tema livre oral no 36 Congresso de 2002.

Artigo recebido em 07 de fevereiro de 2003. Artigo aceito em 16 de outubro de 2003.
} 


\section{INTRODUÇÃO}

Os primeiros anos de vida têm sido considerados críticos no desenvolvimento da audição e da linguagem. 0 diagnóstico e a intervenção precoces são de fundamental importância no desenvolvimento das crianças com alterações auditivas ${ }^{1-4}$

Muito tem se falado da importância de realização de TAN logo após o nascimento ${ }^{1,5-10}$. O s métodos objetivos mais utilizados são as emissões otoacústicas (EO A) e a audiometria de tronco cerebral $(A B R)^{9-14}$.

AsEOA têm sido largamente indicadas para programas de triagem por ser um exame rápido, de fácil aplicação e poder atingir um grande número de sujeitos em um curto espaço de tempo $0^{1,6,10,12,16,17}$.

A ABR tem sido o método de escolha para diagnóstico precoce de disacusias há anos. É um método sensível e específico, porém mais elaborado, exige maior tempo para sua execução, além do custo do equipamento ser elevado $0^{13,18-}$ ${ }^{21}$. Na forma de triagem fica mais difícil sua utilização, principalmente em função do tempo gasto para a realização do exame e em função do custo alto do equipamento. Já se tem proposto 0 uso da ABR automática em programas de triagem auditiva, por este ser mais rápido, porém o custo do equipamento ainda é muito alto para os padrões brasileiros ${ }^{22-25}$.

Tão importante quanto a triagem é o seguimento e o diagnóstico precoce das alterações auditivas das crianças reprovadas na triagem ${ }^{4,26,27,30}$.

Em nosso serviço, as crianças testadas e reprovadas são encaminhadas ao otorrinolaringologista e tendo resolvido possíveis alterações de conduto auditivo externo como cerume ou vérnix, as crianças são retestadas e, se novamente reprovadas, são encaminhadas para a realização de $A B R$.

Os objetivos deste trabalho é verificar quais as alterações mais freqüentes nas $A B R s$ desta população e verificar a adesão da população ao programa de triagem auditiva neonatal.

\section{MATERIAL E MÉTODO}

No período de dezembro de 1999 a março de 2002, foram feitas 5.835 avaliações por EOA no setor de TAN da instituição. Destas, 360 crianças foram encaminhadas ao otorrinolaringologista para avaliação otológica e depois para novo exame por EOA. Após reteste, 128 foram encaminhados para $A B R$, e destas, 75 compareceram para exame.

Analisamos as ABRs de 75 crianças que compareceram para exame após serem reprovadas na TAN por EOA.

A EO A foi realizada em sala acusticamente tratada, utilizando o equipamento ILO 2.920 todynamics Ltda.

A ABR foi realizada sob sono natural ou, se necessário, sob sedação com hidrato de cloral $14 \%$ 0,5ml/3Kg de peso. Utilizou-se o equipamento Smart EP da Inteligent Hearing System, de 2 canais.
Parâmetros utilizados: low pass $1500 \mathrm{~Hz}$, High pass $100 \mathrm{~Hz}$; estímulo click de rarefação; 1024 estímulos a 17.7 estímulos por segundo; fone de inserção ou vibrador ósseo nos casos de microtia ${ }^{19}$.

Foi considerado limiar eletrofisiológico auditivo normal a intensidade de $30 \mathrm{~dB}(\mathrm{HL})$ corrrespondente a $15 \mathrm{~dB}$ limiar psicoacústico. Perda auditiva leve, limiares entre 40 e 50 $\mathrm{dB}(\mathrm{HL})$; perda auditiva moderada, limiares entre 60 e $70 \mathrm{~dB}$ $(\mathrm{HL})$; severa, limiares entre 80 e $90 \mathrm{~dB}(\mathrm{HL})$, e profunda, limiares acima de $90 \mathrm{~dB}(\mathrm{HL})$.

Analisamos através do ABR o limiar eletrofisiológico de cada orelha. A seguir, analisamos a latência da onda $V$ sempre que possível $30 \mathrm{~dB}$ acima do limiar encontrado para cada orelha, mas em limiares acima de $70 \mathrm{~dB}$ a latência foi obtida em intensidade maior de 20, 10 ou no próprio limiar, pois a intensidade máxima do aparelho é de $100 \mathrm{~dB}(\mathrm{HL})$.

Não foram avaliados neste estudo a amplitude da onda $V$ e a latência-amplitude das demais ondas.

As crianças com alterações no ABR foram orientadas a retornar para novo exame após 2 meses.

Este trabalho abordará o primeiro exame realizado. Outro estudo posteriormente avaliará o segundo $A B R$ realizado.

\section{RESULTADOS}

O ABR foi realizado em 75 crianças (150 orelhas).

O Gráfico 1 mostra a distribuição da população por idade e por sexo.

O limiar eletrofisiológico auditivo das orelhas testadas, por número de orelhas está disposto na Tabela 1.

A Tabela 2 mostra o comportamento da onda $V$ no que diz respeito a sua latência, nos diferentes grupos de idade (menor de 30 dias, 1 a 3 meses incompletos, 3 meses a 6 meses incompletos, 6 meses a 9 meses incompletos, 9 meses a 1 ano.

Verificamos que a maior parte das crianças avaliadas apresentou $A B R$ dentro dos padrões normais. A maioria das orelhas testadas ( 61 orelhas, ou 40,67\%) apresentou limiar eletrofisiológico em $30 \mathrm{~dB}(\mathrm{HL})$. Em 55 orelhas (36\%) encontramos perda auditiva leve, em 22 (14,6\%) perda auditiva moderada, em 6 (4\%) perda auditiva severa e em 6 orelhas (4\%) profunda.

$A$ avaliação da latência da onda $V$ mostrou-se aumentada em 64 orelhas $(42,67 \%)$, podendo sugerir alteração condutiva ou retrococlear. Uma porcentagem das crianças (10\%) já apresentaram latências esperadas nos adultos. Sete orelhas $(4,67 \%)$ não apresentaram resposta.

\section{DISCUSSÃO}

A audição é fundamental para aquisição e desenvolvimento de fala e linguagem. Estudos comprovam que a 


\section{Pacientes submetidos a ABR}

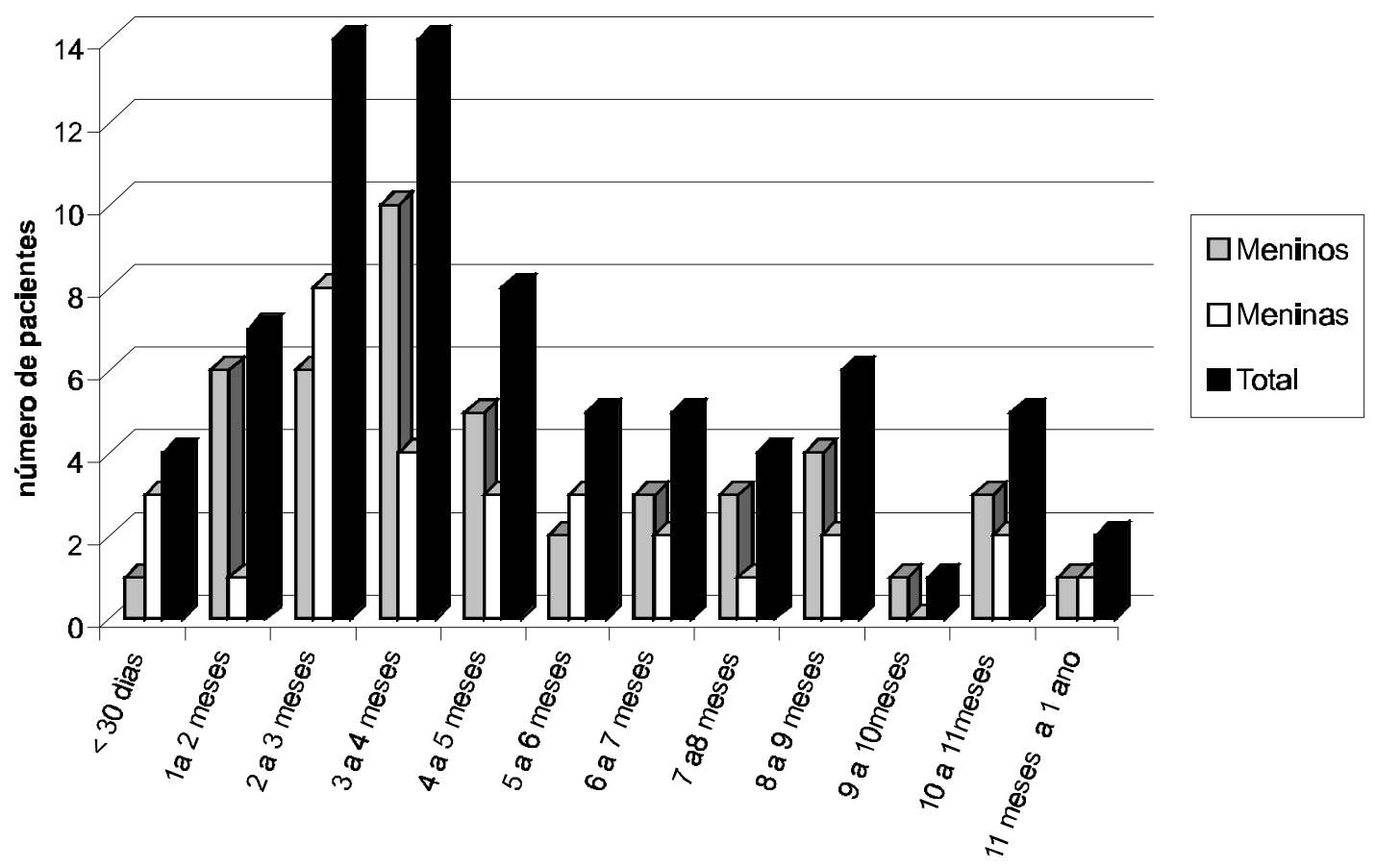

idade

Gráfico 1. Distribuição das crianças submetidas a $A B R$, por idade e por sexo.

Tabela 1. Limiares eletrofisiológicos obtidos, por orelha direita e esquerda.

\begin{tabular}{lcc}
\hline Intensidade $\mathrm{dB}(\mathrm{HL})$ & Orelha esquerda & Orelha direita \\
\hline 30 & 34 & 27 \\
40 & 15 & 16 \\
50 & 9 & 15 \\
60 & 7 & 4 \\
70 & 3 & 8 \\
80 & 1 & 0 \\
90 & 2 & 1 \\
100 & 1 & 1 \\
ausente & 3 & 3 \\
\hline Total & 75 & 75 \\
\hline
\end{tabular}

detecção de alterações auditivas e a intervenção iniciada até os 6 meses de idade garantem à criança o desenvolvimento da compreensão e da expressão da linguagem, bem como, o seu desenvolvimento social, comparável com as crianças normais da mesma faixa etária2,3. Para tanto, o processo de detecção de alterações auditivas deve começar com a triagem auditiva neonatal acompanhada do diagnós- tico e intervenção precoces. Os primeiros 6 meses de vida são decisivos para o desenvolvimento futuro da criança deficiente auditiva ${ }^{3}$.

Após a triagem auditiva, realizada ainda na maternidade, é necessária a realização de diagnóstico nos casos de falha. É imprescindível que outros exames, como a audiometria de tronco cerebral, imitanciometria e observação do comportamento auditivo, sejam incorporados ao de emissões otoacústicas para que o diagnóstico de deficiência auditiva seja realmente concluído ${ }^{16,28}$.

A cada dia mais profissionais tem se conscientizado da importância do diagnóstico precoce das disacusias para o desenvolvimento global e social das crianças. 0 mesmo ainda não acontece com a população leiga ${ }^{16,24}$.

A TAN em nossa cidade não é um exame obrigatório. Nosso serviço oferece o exame a todas as crianças nascidas no serviço, tanto do atendimento público (SUS) quanto dos particulares e convênios. Grande parte das famílias não requerem o exame ainda na internação, como é o idealizado.

Alguns pais retornam semanas ou meses após o nascimento para a realização do exame, retardando assim a avaliação auditiva da criança. 
Tabela 2. Comportamento da onda $V$ em relação à idade, por número de orelhas. (intervalo de idade aberto a direita).

\begin{tabular}{|c|c|c|c|c|c|}
\hline Orelhas com: & Menor 1 mês & 1 a 3 meses & 3 a 6 meses & 6 a 9 meses & 9 a 12 meses \\
\hline latências normais para a idade & 6 & 18 & 17 & 16 & 7 \\
\hline Latências esperadas para adultos & 0 & 7 & 2 & 3 & 3 \\
\hline Total de orelhas & 8 & 42 & 54 & 30 & 16 \\
\hline
\end{tabular}

Outros pais são orientad os a retornar ao serviço para um segundo exame quando, devido à presença de vérnix, o primeiro exame falha. Muitas crianças não retornam. Também, nem todas as crianças encaminhadas ao otorrinolaringologista são levadas a ele. Outras comparecem à consulta, mas não retornam para o reteste. Até mesmo para a realização de $A B R$ muitas crianças encaminhadas não comparecem. Todos estes escapes de pacientes explicam a realização de tantos $A B R$ em idades mais avançadas.

Verificamos que o ABR mostrou-se normal em grande parte das crianças que tinham sido reprovadas nas

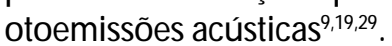

$A$ alteração mais encontrada no $A B R$ foi o aumento da latência da onda $V$, que pode corresponder ou ao retardo da condução elétrica por processo de mielinização ainda em desenvolvimento ${ }^{18,27}$, uma vez que o processamento da informação acústica se equipara ao do adulto por volta dos dois anos e seis meses, enquanto que a onda $V$ está em processo de maturação até os dois anos de idade, ou à alteração retrococlear ou condutiva ${ }^{19}$. Geralmente a alteração condutiva mais encontrada nesta faixa etária é alteração condutiva por secreção em ouvido médio ${ }^{28}$. Isto deve ser confirmado com a realização de timpanometria e, se presente, deve ser logo tratado.

0 acompanhamento destas crianças deve prosseguir até conclusão do caso ${ }^{2,3}$.

\section{CONCLUSÃO}

1- Muitos pacientes se perdem durante a execução do protocolo de triagem auditiva, retardando ou impossibilitando o diagnóstico correto e precoce.

2- A maior parte das crianças rep rovadas nas otoemissões acústicas têm limiar eletrofisiológico auditivo normal.

3- A alteração mais freqüente no ABR foi o aumento da latência da onda V.

\section{REFERÊNCIASBIBUOGRÁFICAS}

1. Garcia CFD, Isaac ML, Oliveira JAA. Emissão otoacústica evocada transitória: instrumento para detecção precoce de alterações auditivas em recém-nascidos a termo e pré-termo. Rev Bras Otorrinolaringol 2002; 68(3): 344-52.
2. Yoshinaga-Itano C. Efficacy of early identification and early intervention. Seminars in hearing 1995; 16(2):115-23.

3. Yoshinaga-Itano C, Sedey AL, Coulter DK, Mehl AL. Language of early and later identified children with hearing loss. 1998; 102(5):1161-71.

4. Serbetcioglu MB. Critical learning period for speech acquisition and screening techiniques in early detection of hearing impairment. Turk J Pediat 2001; 43(2):18-32.

5. Bailey HD, Bower C, Krishnaswamy J, Coates HL. Newborn hearing screening in Western Australia. Med J Aust 2002; 177(4):180-5.

6. Bonfil SP, Narcy P. Auditory screening of infants using evoked otoacoustic emissions. Audiology in practice 1989; 3:4-6.

7. Kaldestad RH, Wingaard L, Hanse TW. Screening for congenital hearing loss - a pilot project. Tedsskr Nor Laegeforen 2002; 122(22): 2190-3.

8. Lin HC, Shu MT, Chang KC, Bruna SM. A universal newborn hearing screening program Taiwan. In J Pediatr Otorhinolaryngol 2002; 63(3):209-18.

9. Quimonez RE, Rodriguez Q. Comparison of neonatal hearing screening devices. PR Health Sci J 2001; 20(4):361-5.

10. Watkin PM. Neonatal screening for hearing impairment. Semin Neonatol 2001; 6(6):501-9.

11. Almenar LA, Tapia TMC, Fernandez P, Moro SM. A combined neonatal hearing screening protocol. An Esp Pediatr 2002; 57(1):559.

12. Andersen SH, Andersen J, Andersen R, Sponheim L. Universal neonatal hearing screening of infants with otoacoustic emissions. Tedsskr Nor Laegeforen 2002; 122(22):2187-9.

13. Chiang MC, Chou YH, Wang PJ. Auditory brainstem evoked potentials in healthy full-term and pre-term infants. Chang Gung Med J 2001; 24(9):557-62.

14. Diez-Delgado RJ, Espin GJ, Lendinez MF, Ortega MMA, Arcos MJ, Lopez MJ. Hearing screening with evoked otoacoustic emission in the neonatal period are logistically a economically feasible. An Es Pediatr 2002; 57(2): 157-62.

15. Balkany T, Telischi FT, McCoy MJ, Lonsbury-Martin BL, Martin GK. Otoacoustic emissions in otologic practice. Am J Otol 1994; 15(1): 29-38.

16. Keefe DH, Zhao F, Neely ST, Gorga MP, Vohr BR. Ear canal acoustic admittance and reflectance effects in human neonates. I. Predictions of otoacoustic emission and auditory brainstem response. J Acoust Soc Am 2003; 113(1):389-406.

17. Veja C, Alvarez SMY. Otoacoustic emissions screening as a early identification of hearing loss as a early identification of hearing loss in newborns. Acta Otorrinolaringol Esp 2001; 52(4): 273-8.

18. Gupta AK, Anand NK. Brainstem evoked response audiometry in neonates. Indian Pediatr 1990; 27(9):1007-9.

19. Hood LJ. Clinical Applications of the auditory brainstem response. San Diego, London: Singular publishing group.

20. Olusanya B. Early detection of hearing impairment in a developing country: what options. Audiology 2001; 40(3):141-7.

21. Burkard RF, Sims D. The human auditory brainstem response to high click rates: aging effects. Am J Audiol 2001; 10(2):53-61. 
22. Boshuizen HC, Van Derlem GJ, Kauffman BMA, Van Zanten GA, Oudesleys MM. Costs of different strategies for neonatal hearing screening: a modelling aproach. Arch Dis Child Fetal Neonatal Ed 1999; 85(3):177-81.

23. Schonweiler R, Tioutou E, Tolloczko R, Pankau R. Hearing screening with automatic evaluation of TEOAE and a new method of automatic evaluation of early auditory evoked potential. Optimization and field trial. HNO 2002; 50(7):649-56.

24. Van Straaten HL, Hille ET, Kok JH, Verkerk PH. Implementation of a nation-wide automated auditory braisntem response hearing screening programme in neonatal intensive care units. Acta Paediatr 2003; 92(3):332-8.

25. Yoshida S, Orihara H, Tanino T, Oshima T. Neonatal auditory screening with automated ABR. Nippon Jibunkoka Gakkai Kaiho 2002; 105(7):804-11
26. Yoon PJ, Price M, Gallagher K, Fleisher B, Messner AH. The need for long-term audiologic follow up of neonatal intensive care unit (NICU) graduates. International journal of Pediatric Otorhinolaryngology 2003; 6(4):353-7.

27. Tibusseck $D$, Meister $H$. Hearing loss in early infancy affects maturation of the auditory pathway. Dev med Child Neurol 2002; 44(2): 123-9.

28. Priner $R$, Freeman $S$, Perez $R$, Sohmer $H$. The neonate has a temporary condutive hearing loss due to fluid in the middle ear. Audiol Neurootol 2003; 8(2):100-10.

29. Mehl AL, Thomson V. The Colorado newborn hearing screening project, 1992-1999 on the thereshold of effective population based universal newborn hearing screening. Pediatrics 2002; 109(1):E7.

30. Cacace AT, Pinheiro JM. Relantionships between otoacoustic emissions and auditory brainstem response in neonates and young children: a correlation and factor analytical study. Laryngoscope 2002; 112(1):156-67. 